\title{
Designing a Nasal Prosthesis using CAD-RP Technology
}

\author{
R Vanitha, K Ramkumar, G Rajtilak, V Rajasekar
}

\begin{abstract}
A 37-year-old female patient reported to the hospital with a nasal defect due to carcinoma. She was previously restored with nasal prostheses, but was not satisfied with its cosmetic appeal. A computerized tomographic (CT) scan of the defect area was made and converted into 3- dimensional (3D) digital data using dedicated medical imaging software. From the 3D image, measurements of the defect were calculated and compared with various nasal fossa measurements available in the digital database. A 3D nose model which had measurements that closely matched the defect area was extracted and superimposed on the defect area and margins adjusted. The data files were then sent for rapid prototyping (RP). A R P model was fabricated which was duplicated in wax and processed. The final result was a nasal prosthesis that conformed well to the patients' face and was also esthetically acceptable. The main advantage of computer-aided designing (CAD)-RP is that it allows trying various nasal forms on the patients face within few hours. This saves chair time, eliminates the impression step and provides patient and dentist an option of variety.
\end{abstract}

Keywords: Computer-aided designing, Rapid prototyping, Fused deposition method, Computerized tomographic scan, Maxillofacial prosthesis.

How to cite this article: Vanitha R, Ramkumar K, Rajtilak G, Rajasekar V. Designing a Nasal Prosthesis using CAD-RP Technology. IntJ Prosthodont Restor Dent 2012;2(3):108-112.

\section{Source of support $\mathrm{Nil}$}

Conflict of interest: None

\section{INTRODUCTION}

Face gives us unique identity and recognition in this society. It is through the face we communicate with the outer world. A ny damage to the face or its parts due to carcinoma or trauma is not well tolerated. With the loss of facial parts like eye, ear, nose, etc. there is not only functional damage but also psychological damage thereby affecting the total quality of life. ${ }^{1-3}$ Hence, such patients should be well rehabilitated and their defects restored to the maximum. It is not always possible to restore facial defects only by surgical means. Surgery along with prosthesis or in some conditions only prosthesis can give satisfying results. ${ }^{4}$

$M$ ajority of nasal defects are secondary to treatment of neoplasms, occasionally, defects secondary to trauma are seen. ${ }^{3}$ Surgery as a means to restore complete nasal defects is difficult if not impossible owing to lack of cartilage to support the nose. ${ }^{1,4}$ Prosthesis can be given to accomplish the goal of providing near normal appearance for such patients. ${ }^{3}$ Common methods used for fabricating nasal prosthesis include sculpting a pattern from a mass of wax or clay or making a cast of the patient's nose prior to surgery and duplicating it. This former method is time consuming and requires increased number of patient visits and a talented sculptor. The main drawback in the latter method is that patients come for prosthesis only after surgery is done. All these pose hurdles in fabricating a satisfying nasal prosthesis.

With the entry of computer-aided designing/computeraided manufacturing ( $C A D / C A M)$ technology into reconstructive dentistry, fabrication of extraoral prosthesis has been taken to a higher level in terms of contour replication and finesse detail reproduction. ${ }^{2,5-7}$ CAD has made it possible for us maxillofacial prosthodontists to design and fabricate precise extraoral prostheses that would otherwise require the skill of a finely talented sculptor.

Rapid prototyping (RP) is one of the two types of CAM , the other being computerized numerically controlled (CNC) milling. RP is a generic name given to related technologies that fabricate physical object directly from CAD data. V arious RP technologies available are stereolithography, fused deposition method (FDM), laminated object manufacturing and selective laser sintering. A mong these, the most common technologies used for medical modeling are stereolithography and FDM ${ }^{8}$

This article describes a CAD-RP technology for fabricating custom nasal prosthesis based on the type of nose and its desired size. The RP technology used for obtaining a physical model was FDM . FDM is an additive procedure where the prototype or the solid physical model is fabricated by depositing thermoplastic acrylonitrile butadiene styrene (ABS) resin in a layer by layer fashion according to the data fed to the machine. ${ }^{8}$

\section{CASE REPORT}

A 37-year-old, female patient reported to the Department of Prosthodontics, Government Dental College and Hospital, Chennai, with a nasal defect. The nasal defect was a postsurgical one following total rhinectomy for squamous cell carcinoma. The defect extended superiorly to the root and inferiorly to the base of nose and laterally short of the nasolabial fold (Fig. 1). She had previously been restored with three different nasal prostheses in the past 5 years (Fig. 2). Poor cosmetic appeal of all of the previous prostheses was the main reason for her seeking a new prosthesis. The patient had no siblings or a presurgical photograph that would have hel ped in sculpting a wax model of the prosthesis. Therefore it was decided to use CA D-RP technology to design the nasal prosthesis. The option of 


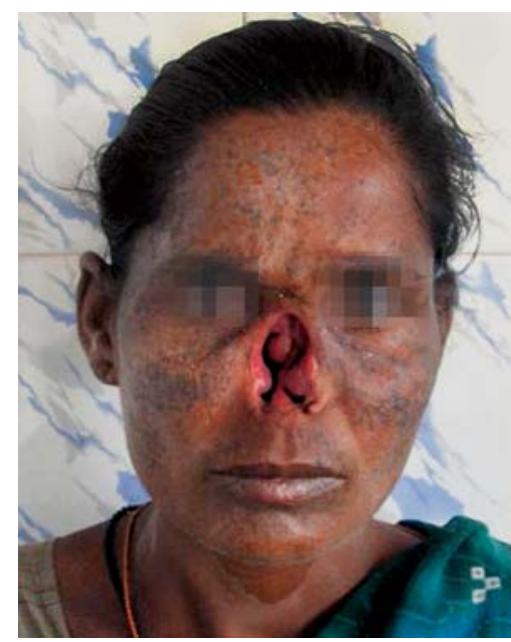

Fig. 1: Patient with nasal defect

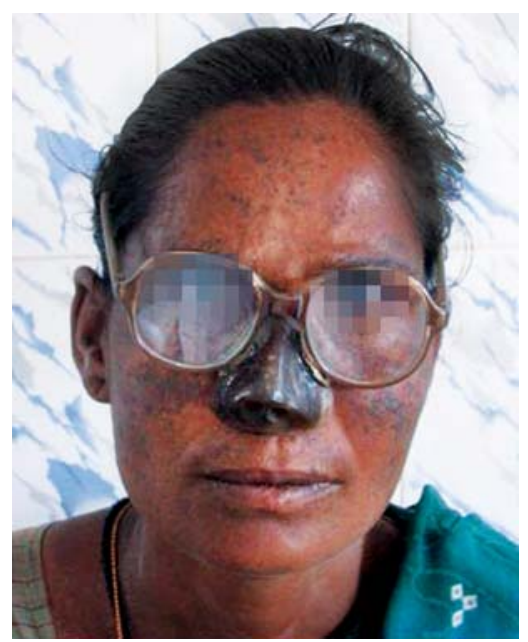

Fig. 2: Patient with previous prosthesis

implants was provided to the patient for retention of the prosthesis but as she did not want an invasive surgical procedure and was habituated to the spectacles, she preferred the latter method of retention.
A contiguous spiral computerized tomographic (CT) scan of the patients face was made at intervals of $1.25 \mathrm{~mm}$ thickness. The patients head was stabilized and a scan from the supraorbital ridge to the junction of the upper and lower lip was made. The data acquired was converted to digital imaging and communications in medicine (DICOM ) images and downloaded in a disk (Fig. 3). The images were stored in $x, y, z$ format. DICOM was used because of its simplicity, best output and compatibility with other CAD packages. Using 3D Doctor Software, data was processed and a 3D digital model of face obtained (Fig. 4). Using this software, soft tissue contour was also displayed. This image could be rotated mediolaterally, anteroposteriorly and tilted to any angle in any direction. The projection of the face from any angle could thus be view ed. This is important as this facility allows viewing the profile of the patient.

$M$ idline of the face was used as the axis of symmetry. The defect was measured in height and width (Fig. 5). These measurements were compared with the nose measurements available in the digital database (Fig. 6). The values of those digital noses that matched the defect measurements were chosen and superimposed on the defect area. The digital model that suited the most with the patients face with respect to both the measurements and cosmetic appeal was chosen. It was custom modified and the margins smoothed to perfection (Fig. 7). This was done using free form software. Once the digital nasal model was perfected, it was transferred into a data file compatible for RP procedure. U sing FDM , a solid physical model (nasal prototype) wad obtained (Fig. 8). The obtained plastic physical model was duplicated in wax using vinyl polysiloxane, ${ }^{2}$ tried on the patients' face and margins checked. This wax nose model was invested in a manner to construct a two-piece mold; dewaxed, basic shade determined and processed using RTV silicone (Fig. 9).
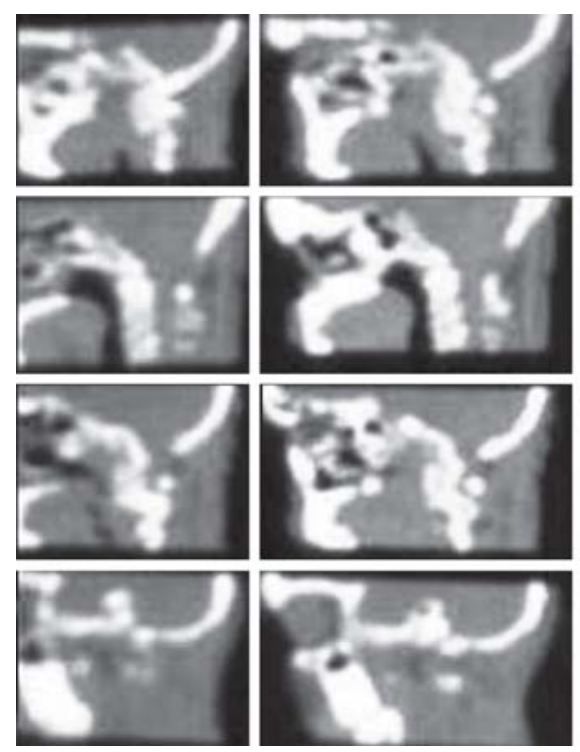
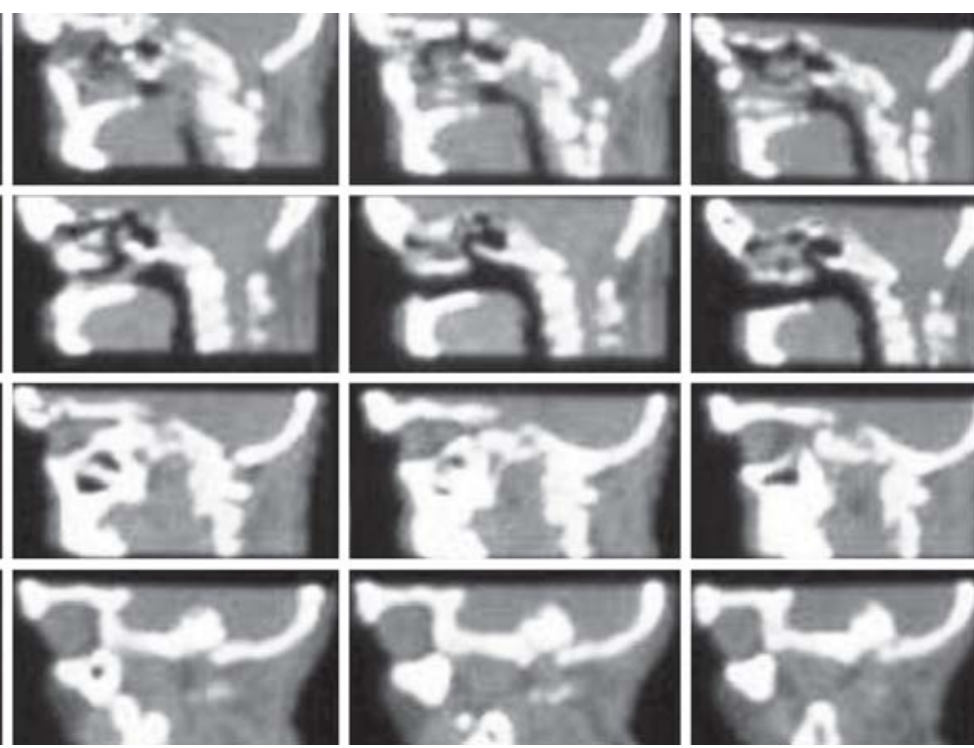

Fig. 3: CT scan data
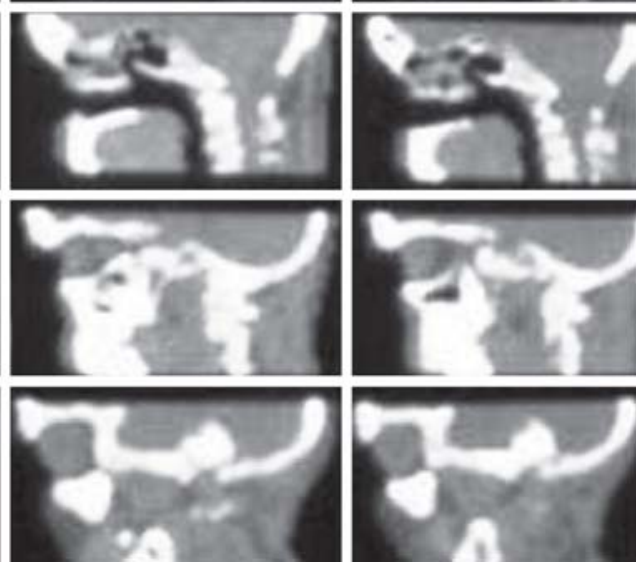


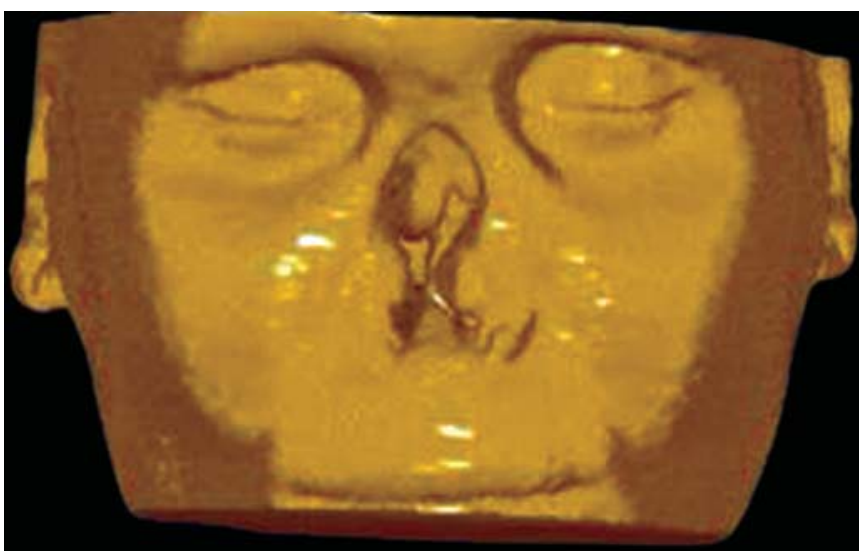

Fig. 4: 3D digital model of patients' face

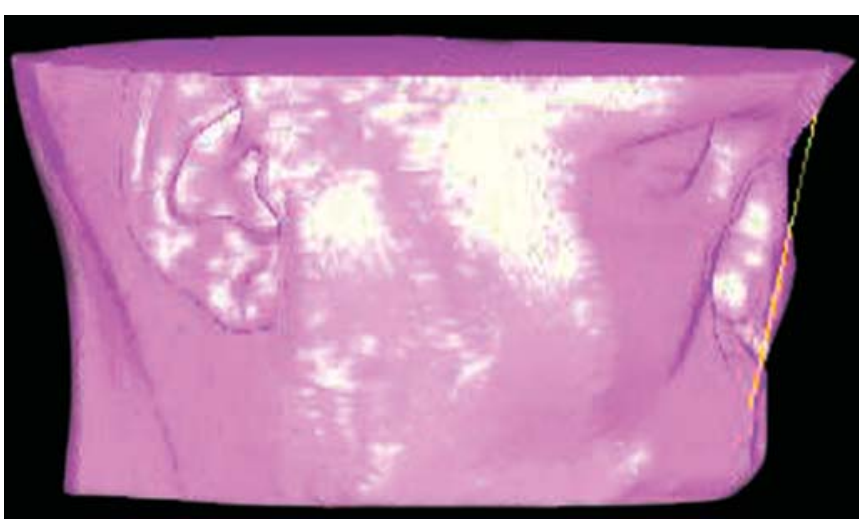

Fig. 5: Defect measured

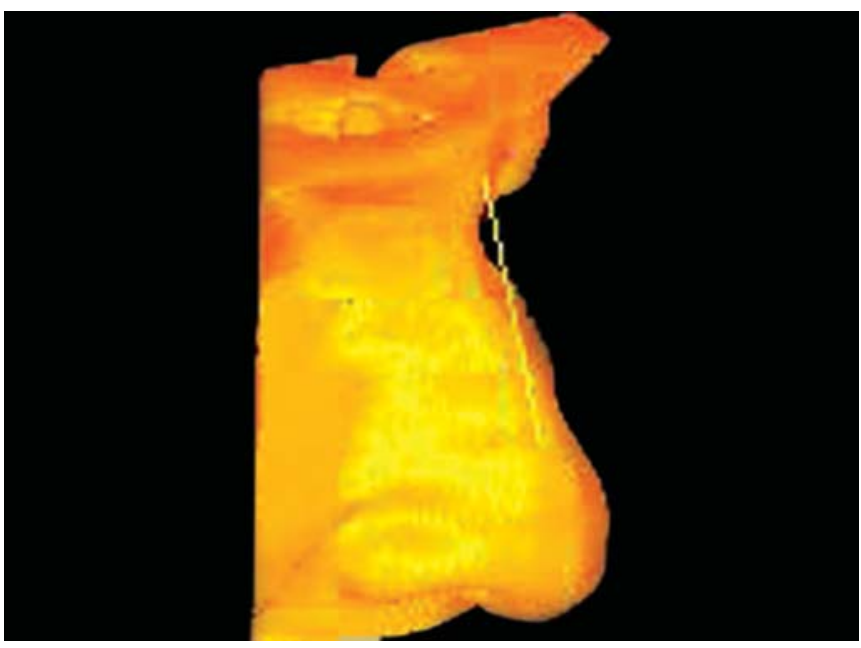

Fig. 6: 3D model of nose from digital data base

The nasal prosthesis was glued to eye glass frame which served as a retention aid and given to the patient (Fig. 10) at which time detailed instructions regarding care and use were provided.

\section{DISCUSSION}

Loss of any part of the human body especially one from the face can be a very traumatic experience. N ose among them requires special mention as it is located in the prominent

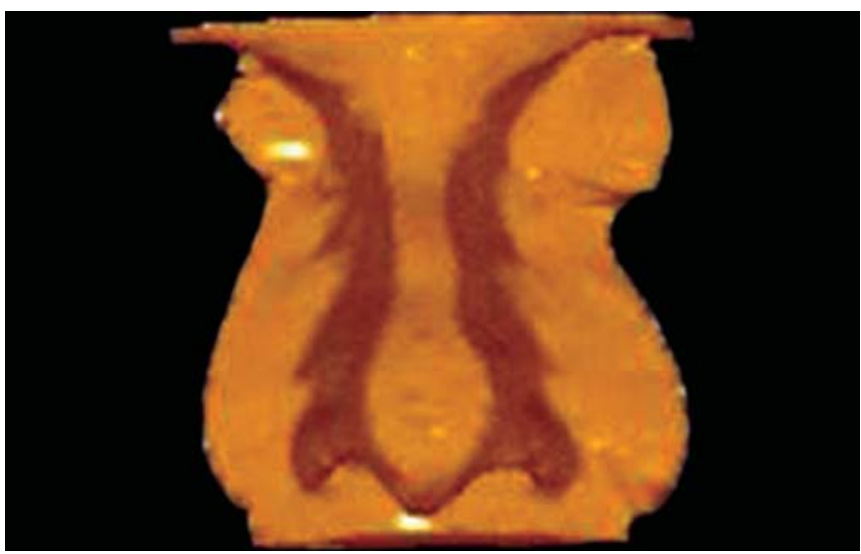

Fig. 7: S uperimposed digital model

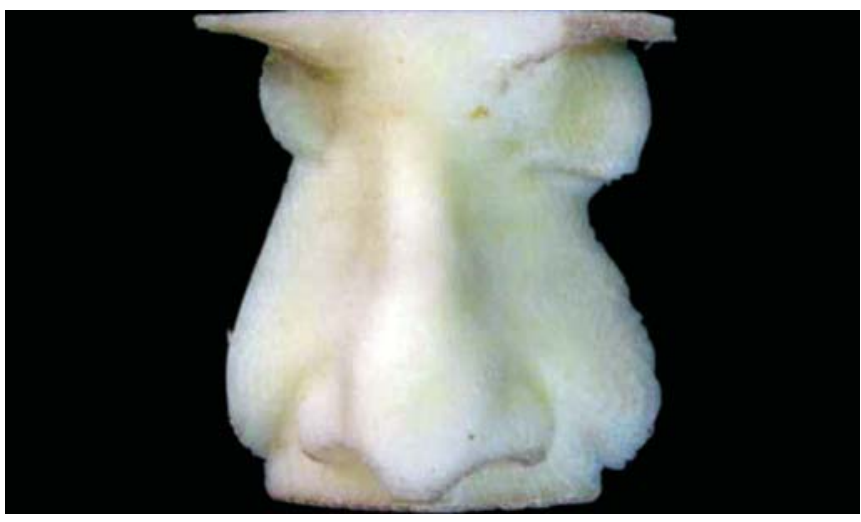

Fig. 8: Physical model of the nose in ABS plastic

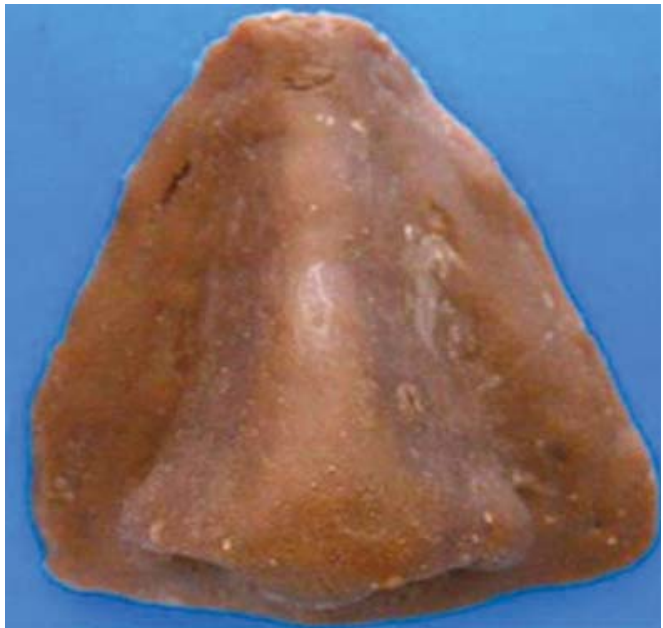

Fig. 9: Processed prosthesis

central part of the face. M oreover, it is one such structure that cannot be hid casually like eyes (using tinted glasses) and ears (behind the hair). Therefore, a prosthesis that exactly replaces that of the patients' normal nose is very crucial to the success of rehabilitation. A lthough it can be stated that unlike the eyes and ears, nose does not have any counterpart and therefore easy to reconstruct as there is no possibility of comparing it with the normal part, ${ }^{9}$ it is very important to consider the fact that unless a previous 


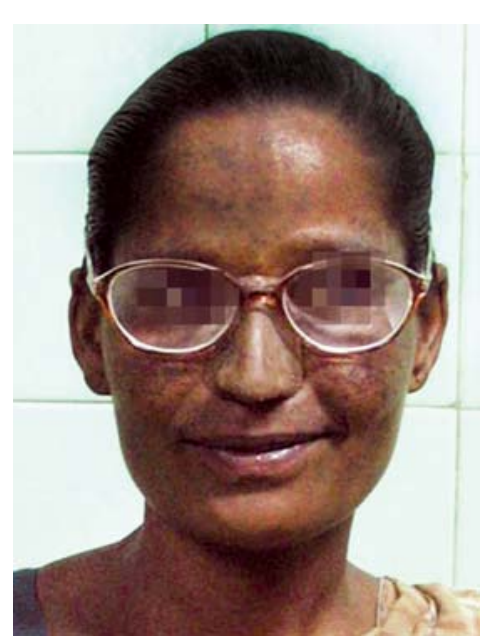

Fig. 10: Patient with new prosthesis

photograph or a cast of the normal structure is present, this is very difficult. ${ }^{5} \mathrm{M}$ oreover, if the patient requires prosthesis that does not simulate the normal nose, it can be an uphill task.

The standard procedure, 'donor technique' involves taking an impression of the nose of a person who matches the patients' morphological feature (e.g. sibling, relative) and duplicating it in wax. This is custom modified and processed. ${ }^{9}$ The methods most commonly used for retaining nasal prosthesis include involving anatomical undercuts if available, medical adhesives, mechanical support (e.g. eyeglasses) ${ }_{10}^{10}$ prosthetic connections to endosseous implants ${ }^{10,11}$ and attachment to maxillary obturators. ${ }^{10,12}$ The medical adhesives used to retain prostheses may irritate soft tissues that contact the prosthesis and may damage the thin margins of the prosthesis when it is removed for maintenance and cleaning. A n eyeglasses-supported nasal prosthesis system may result in displacement, with an opening at the margins of the prosthesis. For definitive rehabilitation, osseointegrated craniofacial implants (two positioned in the premaxilla in the nasal floor and one in the glabella, if necessary) and a metal support bar to stabilize the prosthesis are best for anchoring a nasal prosthesis to the skull. ${ }^{13}$

A Iternatively, nose pattern can be directly sculpted on the cast of the patient and tried. This involves usage of anatomic contours as reference augmented by preoperative photograph supplied by the patient. ${ }^{10,14-16} \mathrm{~V}$ ery rarely, a defect free presurgical cast of the patient along with a photograph, if available can serve as an excellent model for duplicating the nose of the patient. ${ }^{14}$ However, this procedure did not allow the choice of using various types of nasal structure. This is because it is very time consuming to compare infinite wax models of the nasal structure. M oreover, such library of wax models of noses is not readily available.
The introduction of CT and M RI with 3D representation of human anatomy has opened up a new door for alternative methods of designing an anatomical structure. CAD allows precise designing of the form in terms of both contour and texture and CAM allows precise reproduction of the designed model. So this recent technology was adopted for the fabrication of the nasal prosthesis. The advantages of CAD-RP are manifold:

a. With a proper digital library, various shapes of the nasal structure could be superimposed on the digital model and the required one opted in a matter of a few hours. ${ }^{13,17}$

b. For paired structures (e.g. eyes, ears), duplication to the exact dimension and mirroring can be done. . $^{2,6,7}$

c. Using RP, complex internal forms as those present in the external ear can be reproduced with precision.

d. Eliminates the need for an impression procedure.

e. Eliminates the dependency on close relatives for the nasal impression.

f. The digital model and the plastic prototype can be preserved. This is important as replacement of the prosthesis is required once in few years following discoloration, change in fit, tearing, aging and general wear. The plastic prototype also allows multiple pouring for shade matching purposes. ${ }^{2}$

g. They do not require a skilled anaplastologist for sculpting the clay model of the defect area (especially the ears).

h. The possibility of 3D visualization and easy virtual changes (type of nose, dimensions, position on face) ensure optimization of the whole process prior the manufacturing. ${ }^{17,18}$ In addition, it allows the feedback of the patient to be incorporated.

The disadvantages of CAD-RP are:

a. Cost factor. ${ }^{7}$

b. Radiation dose: Several articles have reported various techniques to reduce the radiation dose. . $^{2,5,6,13}$

c. Requires equipment and computational skills of the CAD-CAM methods. ${ }^{18}$

\section{CONCLUSION}

Several authors have attempted to design facial prosthesis (ear, eyes and nose) using CAD-CA M technology. 2,5-7,13,17-20 This has helped in reducing time and increasing the quality of the final product. While conventional procedures have to depend on the patients siblings or close relatives or prefabricated casts along with photographs which may not be available at all times, CAD offers the edge of not having to depend on any of these. In addition it offers limitless shapes and sizes of digitized nose that can be checked 
virtually on the patient's digitized face. ${ }^{13,17}$ This can be helpful if the patient had not been pleased with his natural nose shape. Although it may seem to be costly in terms of money, it does reduce patient's number of visits and chairside time. Recent article has shown how CAD prosthesis can be more economical on finance and time than a manually built prosthesis. ${ }^{13}$

This article presents a technique for designing nasal prosthesis using CAD-RP technology which was retained using spectacles. Nasal prosthesis and spectacles both designed and fabricated using CA D-RP technology has also been reported. ${ }^{13}$ Given the advantages, in years to come CAD-RP will establish itself as a standard treatment procedure.

\section{ACKNOWLEDGMENTS}

The authors thank Dr Jayanti Parthasarathy, MS, for her skilled guidance in computer designing (CA D) of the nasal prosthesis.

\section{REFERENCES}

1. Taylor TD. Clinical maxillofacial prosthetics. Chicago: Quintessence 2000;1-14.

2. Ciocca L, Scotti R. CAD-CA M generated ear cast by means of a laser scanner and rapid prototyping machine. J Prosthet D ent 2004:92:591-95.

3. Beumer J, Curtis TA, M arunick M T. M axillofacial rehabilitation: Prosthodontic and surgical considerations (2nd ed). St Louis: Ishiyaku EuroA merica 1996;402.

4. Beumer J, Curtis TA, M arunick M T. M axillofacial rehabilitation: Prosthodontic and surgical considerations (2nd ed). St Louis: Ishiyaku EuroA merica 1996;378.

5. Reitemeier B, Notni G, Heinze M, Schone C, Schmidt A, Fichtner D. Optical modeling of extraoral defects. J Prosthet Dent 2004;91:80-84

6. Mardini M A, Ercoli C, Graser GN . A technique to produce a mirror-image wax pattern of an ear using rapid prototyping technology. J Prosthet Dent 2005;94:195-98.

7. Penkner K, Santler G, Mayer W, Pierer G, Lorenzoni M . Fabricating auricular prostheses using three-dimensional soft tissue models. J Prosthet Dent 1999;82:482-84.

8. Winder J, B ibb R. M edical rapid prototyping technologies: State of the art and current limitations for application in oral and maxillofacial surgery. J Oral M axillofac Surg 2005;63:1006-15.

9. Taylor TD. Clinical maxillofacial prosthetics. Chicago: Quintessence 2000;239-40.

10. Secilmis A, Ozturk AN. Nasal prosthesis rehabilitation after partial rhinectomy: A clinical report. Eur J Dent 2007;1(2): 115-18.
11. B rånemark $\mathrm{PI}$, Tolman $\mathrm{DE}$. Osseointegration in craniofacial reconstruction. Carol Stream, Illinois: Q uintessence Publishing Co, Inc., 1998;93-208.

12. Guttal SS, Patil NP, Shetye AD. Prosthetic rehabilitation of a midfacial defect resulting from lethal midline granuloma: $A$ clinical report. J Oral Rehabil 2006;33:863-67.

13. Ciocca L, Fantini M , Crescenzio FD, Persiani F, Scotti R. N ew protocol for construction of eyeglasses-supported provisional nasal prosthesis using CA D/CAM techniques. JRRD 2010;47(7): 595-604.

14. Beumer J, Curtis TA , M arunick M T. M axillofacial rehabilitation: Prosthodontic and surgical considerations (2nd ed). St Louis: Ishiyaku EuroA merica 1996;405-06.

15. Jain S, Maru K, Shukla J, Vyas A, Pillai R, Jain P. Nasal prosthesis rehabilitation: A case report. J Indian Prosthodont Soc 2011;11(4);265-69.

16. Toljanic JA, Lee J, Bedard JF. Temporary nasal prosthesis rehabilitation: A clinical report. J Prosthet Dent 1999;82: 384-86.

17. Rosicky J, Paravan M, Kempna A, Palousek, D.CAD-CAM design and fabrication of a custom-made nasal prosthesis for total rhinectomy. Paris, France: Presented at the IAA 24th A nnual Conference 2009 N ovember; 12-15.

18. Ciocca L, Bacci G, Mingucci R, Scotti R. CAD-CAM construction of a provisional nasal prosthesis after ablative tumour surgery of the nose: A pilot case. Eur J Cancer Care 2009;18(1):97-101.

19. Ciocca L, Maremonti P, Bianchi B, Scotti R. Maxillofacial rehabilitation after rhinectomy using two different treatment options: Clinical reports. J Oral Rehabil 2007;34(4):311-15.

20. Ciocca L, Fantini M, Marchetti C, Scotti R, M onaco C. Immediate facial rehabilitation in cancer patients using CADCAM and rapid prototyping technology: A pilot study. Support Care Cancer 2010;18(6):723-28.

\section{ABOUT THE AUTHORS}

\section{R Vanitha (Corresponding Author)}

Reader, Department of Prosthodontics, RVS Dental College Coimbatore, Tamil Nadu, India, e-mail: doc_vanitha@yahoo.com

\section{K Ramkumar}

A ssistant Professor, Department of Prosthodontics, Government Dental College, Chennai, Tamil Nadu, India

\section{G Rajtilak}

Professor and Head, Department of Prosthodontics, RVS Dental College, Coimbatore, Tamil Nadu, India

\section{Rajasekar}

Senior Lecturer, Department of Prosthodontics, RVS Dental College Coimbatore, Tamil Nadu, India 